Research Article

Human and Medical Genetics

\title{
Genetic variability of blood groups in southern Brazil
}

Gabriela Waskow $^{1 *}$, Mirelen Moura de Oliveira Rodrigues ${ }^{1 *}$, Gabriela Höher ${ }^{1}$, Tor Onsten ${ }^{2}$, Juliana Dal-Ri Lindenau $^{3}$, Marilu Fiegenbaum ${ }^{1,4}$ and Silvana Almeida ${ }^{1,4}$ (C)

${ }^{1}$ Universidade Federal de Ciências da Saúde de Porto Alegre (UFCSPA), Programa de Pós-Graduação em Biociências, Porto Alegre, RS, Brazil.

${ }^{2}$ Hospital de Clínicas de Porto Alegre (HCPA), Porto Alegre, RS, Brazil.

${ }^{3}$ Universidade Federal de Santa Catarina (UFSC), Departamento de Biologia Celular, Embriologia e Genética, Florianópolis, SC, Brazil.

${ }^{4}$ Universidade Federal de Ciências da Saúde de Porto Alegre (UFCSPA), Departamento de Ciências Básicas da Saúde, Porto Alegre, RS, Brazil.

\begin{abstract}
We evaluated genetic variability among the blood groups Kell (c.578C $>T$ and c.1790T $>C$ ), Kidd $(c .838 A>G)$, Duffy $(c .125 A>G, c .265 C>T$ and c.1-67T > C), Diego $(c .2561 C>T)$, MNS $(c .143 T>C)$ and Rh $(c .676 G>C)$ in Rio Grande do Sul in southern Brazil. Genetic profiling from 382 volunteer blood donors was performed through allelic discrimination assays using a hydrolysis probe (TaqMan®) with a real-time PCR system. The sample was divided into two groups: Euro-Brazilian and Afro-Brazilian. A comparison with studies from other regions of Brazil and the 1000 Genomes Database showed significant differences for almost all polymorphisms evaluated in our population. Population differentiation between the Euro- and Afro-Brazilian groups was low ( $F_{\mathrm{ST}}$ value 0.055$)$. However, when each locus was evaluated individually, $K E L^{*} 06$ and $F Y^{*} 02 N .01$ allele frequencies were significantly higher in the Afro-Brazilian group than in the Euro-Brazilian group. Ethnic classification that uses phenotypic criteria to find blood units with rare antigens may be important when there is a need to detect blood units with an absence of Duffy antigens. There is also a greater probability of finding donors in the Afro-Brazilian group. Taken together, the data indicate strong European and African contributions to the gene pool, with intense admixture.
\end{abstract}

Keywords: Genotyping techniques, blood group antigens, genetic variation, transfusion reaction, genetic polymorphism.

Received: November 5, 2018; Accepted: August 8, 2019.

\section{Introduction}

Blood group systems are characterized by the presence of antigens on red blood cells (RBCs) (Issitt and Anstee, 1998). Currently, 345 blood group antigens are recognized, of which 316 are dispersed among 36 blood group systems (International Society of Blood Transfusion, 2017). Some of these antigens are highly immunogenic, resulting in alloimmunization, hemolytic transfusion reaction (HTR) and hemolytic disease of the fetus and newborn (HDFN) (Anstee, 2009). The main complication directly associated with blood transfusion is HTR, which is mainly induced by the presence of antibodies for blood group antigens (Issitt and Anstee, 1998).

Overall, better characterization of the profiles of blood donors/recipients might increase compatibility and

Send correspondence to Silvana Almeida. Universidade Federal de Ciências da Saúde de Porto Alegre (UFCSPA), Departamento de Ciências Básicas da Saúde, Rua Sarmento Leite, 245, Porto Alegre, RS, Brazil. E-mail: salmeida@ufcspa.edu.br.

*These authors contributed equally to the study. consequently blood transfusion safety. Some rare blood antigens have highly variable frequencies among distinct populations (Pellegrino et al., 2001), which directly impacts transfusion practice, and knowledge of the frequencies of antigens of the main blood groups in each population may help in the search for compatible donors. This is notably true for admixed populations, such as those in Brazil.

Identification of variability in blood groups provides insight into gene ethnic diversity (Pellegrino et al., 2001). Brazil has a territory of continental size, with an area of more than 8 million $\mathrm{km}^{2}$ (Instituto Brasileiro de Geografia e Estatística, 2017). Moreover, in Brazil, interethnic crosses from four continents, Europe, Africa, America and Asia, have formed one of the most heterogeneous populations in the world (Parra et al., 2003; Pena et al., 2011; Durso et al., 2014). Although there are data concerning RBC allelic variability throughout the country (Ribeiro et al., 2009; Baleotti et al., 2011; Credidio et al., 2011; Guelsin et al., 2011; Cruz et al., 2012; Faria et al., 2012; Mota et al., 2012; Arnoni et al., 2013; Piassi et al., 2013; Costa et al., 2016a, 
2016b; Zacarias et al., 2016), such data are not available for Rio Grande do Sul in southern Brazil. In general, these data may improve the searchability and availability of compatible blood units for patients with antibodies to blood group antigens.

Therefore, the aim of this study was to determine the allelic frequencies of polymorphisms in genes in clinically important blood groups, including Kell $(c .578 C>T$ and $c .1790 T>C)$, Kidd $(c .838 A>G)$, Duffy $(c .125 A>G$, $c .265 C>T$ and $c .1-67 T>C)$, Diego $(c .2561 C>T)$, MNS $(c .143 T>C)$, and $\mathrm{Rh}(c .676 G>C)$, in blood donors from a city in southern Brazil. Additionally, the study aimed to evaluate whether ethnic classification might improve the search for rare blood units in a blood center.

\section{Material and Methods}

\section{Sample characterization}

A total sample of 382 regular repetitive voluntary blood donors of both sexes was collected from the Blood Bank of Hospital de Clínicas de Porto Alegre (HCPA), Rio

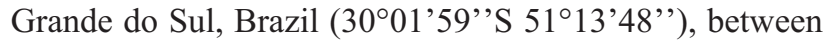
2012 and 2015. The population from southern Brazil is ethnically admixed (Santos, 2002; Flôres et al., 2014); thus, the sample was divided into Euro-Brazilian $(n=334)$ and Afro-Brazilian $(n=48)$ groups. This classification was performed by trained blood bank professionals according to the following phenotypic characteristics: color and texture of hair, skin color in the medial part of the arm, and the shape of the nose and lips (Parra et al., 2003). This is a standard classification used to assist rare phenotypes.
The blood donors agreed to participate through written informed consent. This study was approved by the Ethics Research Committee of the Hospital de Clínicas de Porto Alegre (No: 110418) and the Universidade Federal de Ciências da Saúde de Porto Alegre (UFCSPA) (No: 1829-12).

\section{DNA extraction and genotyping}

Genomic DNA was extracted from peripheral blood leukocytes by a standard salting out procedure (Lahiri and Nurnberger, 1991). The DNA samples were quantified based on optical density at $260 \mathrm{~nm}$ (BioSpec-Nano, Shimadzu, Columbia, MD) and diluted to $10 \mathrm{ng} / \mu \mathrm{L}$.

As listed in Table 1, single nucleotide polymorphisms (SNPs) c.578C > T and c.1790T $>C$ in KEL (Kell, rs8176058 and rs8176038), c.838A $>G$ in $S L C 14 A 1$ (Kidd, rs1058396), c.125A $>G, c .265 C>T$ and c.1-67T $>C$ in $A C K R 1$ (Duffy, rs12075, rs34599082 and rs2814778), c. $2561 C>T$ in $S L C 4 A 1$ (Diego, rs2285644), c.143T $>C$ in GYPB (MNS, rs7683365), and c.676G $>\mathrm{C}$ in RHCE (Rh, rs609320) were analyzed by allelic discrimination using TaqMan 5'-nuclease assays with a real-time PCR system (StepOnePlus, Applied Biosystems, Foster City, CA, USA). The following assays were used: AH8979I, C_25596899_20, C_1727582_10, C_2493442_10, C_11324554_10, C_15769614_10, C_26654865_10, C_34183121_10, and AH5I4HL (ThermoFisher Scientific, Waltham, MA). The reactions were performed with fast thermal cycling conditions with $1 \mathrm{X}$ TaqMan ${ }^{\circledR}$ genotyping master mix, $1 \mathrm{X}$ TaqMan ${ }^{\circledR}$ genotyping assay, $10 \mathrm{ng}$ of DNA and nuclease-free water (final volume $8 \mu \mathrm{L}$ ).

Table 1 - Characterization of blood groups alleles evaluated in present study.

\begin{tabular}{|c|c|c|c|c|c|}
\hline BGS & Gene & Allele & dbSNP & Nucleotide & Amino acid \\
\hline \multirow[t]{2}{*}{ Kell } & $K E L$ & $K E L * 01$ & rs8176058 & c. $578 \mathrm{C}>\mathrm{T}$ & p.Thr193Met \\
\hline & & $K E L * 02$ & & & \\
\hline \multirow[t]{2}{*}{ Kell } & $K E L$ & $K E L * 06$ & rs8176038 & c. $1790 \mathrm{~T}>\mathrm{C}$ & p.Leu597Pro \\
\hline & & $K E L * 07$ & & & \\
\hline \multirow[t]{2}{*}{ Kidd } & SLC14A1 & $J K^{*} 01$ & rs1058396 & c. $838 \mathrm{~A}>\mathrm{G}$ & p.Asn280Asp \\
\hline & & $J K^{*} 02$ & & & \\
\hline \multirow[t]{2}{*}{ Duffy } & $A C K R 1$ & $F Y^{*} 01$ & rs12075 & c. $125 \mathrm{~A}>\mathrm{G}$ & p.Asp42Gly \\
\hline & & $F Y^{*} 02$ & & & \\
\hline Duffy & $A C K R 1$ & $F Y^{*} 02 W .01$ & rs34599082 & c. $265 \mathrm{C}>\mathrm{T}$ & p.Arg89Cys \\
\hline Duffy & $A C K R 1$ & $F Y^{*} 02 N .01$ & rs2814778 & c. $1-67 \mathrm{~T}>\mathrm{C}$ & p.0 \\
\hline \multirow[t]{2}{*}{ Diego } & $S L C 4 A 1$ & $D I^{*} 01$ & rs2285644 & c. $2561 \mathrm{C}>\mathrm{T}$ & p.Pro854Leu \\
\hline & & $D I^{*} 02$ & & & \\
\hline \multirow[t]{2}{*}{ MNS } & $G Y P B$ & $G Y P B * S$ & rs7683365 & c. $143 \mathrm{~T}>\mathrm{C}$ & p.Met $48 \mathrm{Thr}$ \\
\hline & & $G Y P B *^{*}$ & & & \\
\hline \multirow[t]{2}{*}{$\mathrm{Rh}$} & RHCE & $R H C E^{*} E$ & rs609320 & c. $676 \mathrm{G}>\mathrm{C}$ & p.Ala226Pro \\
\hline & & $R H C E^{*} e$ & & & \\
\hline
\end{tabular}

Data obtained from ISBT; (Issitt and Crookston, 1984). BGS = Blood group system. 


\section{Statistical analyses}

A chi-square adjustment test was applied to determine whether the distribution of observed genotype frequencies agreed with those expected under Hardy-Weinberg equilibrium (HWE). We compared the allele frequencies in the present study with data in the 1000 Genomes database (Ensembl GRCh38 - phase III) (African - AFR, European - EUR, East Asian - EAS, South Asian SAS, and Admixed American - AMR) and data for blood donors from other states of Brazil [Santa Catarina - SC (Costa et al., 2016a,b), Paraná - PR-POP1 and PR-POP2 (Guelsin et al., 2011; Zacarias et al., 2016), São Paulo - SP (Ribeiro et al., 2009), Bahia - BA (Costa et al., 2016a,b), and Minas Gerais - MG (Alves et al., 2018)]. Comparison of allelic frequencies was performed using Fisher's exact test with R software in the Rcmdr package (Fox, 2005). A $p$-value $<0.05$ was considered significant.

Genetic distance was determined as $F_{\mathrm{ST}}$ using the Arlequin v.3.5 program (Excoffier and Lischer, 2010), and $95 \%$ confidence intervals were estimated with $\mathrm{R}$ software using the diveRsity package with 3000 bootstraps (Keenan and McGinnity, 2013).

\section{Results}

The distribution of genotype frequencies was in HWE. Minor allele frequencies (MAFs) of the investigated polymorphisms in the Euro- and Afro-Brazilians of our sample, 1000 Genomes database, and data of blood donors from other states of Brazil are shown in Table 2. In our study, $K E L^{*} 06$ and $F Y^{*} 02 N .01$ allele frequencies differed between the Euro- and Afro-Brazilian subgroups ( $p=0.023$ and $p<0.001$, respectively; Table 2 ). When compared to the 1000 Genomes database, the allele frequencies of our Euro-Brazilians were different from those described for AFR, EAS, and SAS populations, except for the $G Y P B * S$ allele in the SAS population and the $K E L^{*} 06$ allele in the EAS and SAS populations. When compared to EUR, the allele frequencies in Euro-Brazilians for $J K^{*} 02(p=0.002)$, $F Y^{*} 02 N .01\left(p<0.001\right.$, ) and $D I^{*} 01(p=0.002)$ variants differed. Comparison with the AMR population also revealed differences in $J K^{*} 02(p<0.001), D I^{*} 01(p<0.001)$, and $R H C E^{*} E(p<0.001)$ variants. Comparison of Euro-Brazilians with blood donors from other regions of Brazil indicated differences in allele frequencies for $F Y^{*} 01$ ( $p<$ $0.001), F Y^{*} 02 N .01(p=0.047)$, and $D I^{*} 01(p=0.014)$ from

Table 2 - Minor allele frequencies of blood groups variants in Euro and Afro-Brazilians from Rio Grande do Sul, 1000 Genomes Database and previous studies performed at Brazil.

\begin{tabular}{|c|c|c|c|c|c|c|c|c|c|}
\hline Sample (n) & $K E L * 01$ & $K E L * 06$ & $J K^{*} 02$ & $F Y * 01$ & $F Y^{*} 02 W .01$ & $F Y^{*} 02 N .01$ & $D I * 01$ & $G Y P B * S$ & $R H C E^{*} E$ \\
\hline Euro-Brazilians (334) & 0.033 & 0.003 & 0.424 & 0.444 & 0.015 & 0.076 & 0.010 & 0.320 & 0.154 \\
\hline Afro-Brazilians (48) & 0.010 & 0.021 & 0.353 & 0.357 & 0.000 & 0.408 & 0.011 & 0.350 & 0.166 \\
\hline$p$ & $n s$ & 0.023 & $n s$ & $n s$ & $n s$ & $<0.001$ & $n s$ & $n s$ & $n s$ \\
\hline $\operatorname{EUR}(503)^{1}$ & 0.038 & $0.000^{\S}$ & $0.501^{¥ \S}$ & 0.398 & 0.013 & $0.006^{¥ \S}$ & $0.000^{*}$ & 0.339 & 0.160 \\
\hline $\operatorname{AFR}(661)^{2}$ & $0.002^{\#}$ & $0.099^{¥ \S}$ & $0.228^{¥ \S}$ & $0.019^{¥ \S}$ & $0.000^{¥ \mathrm{f}}$ & $0.964^{¥ \S}$ & $0.001^{*}$ & $0.185^{¥ \S}$ & $0.080^{¥}$ \\
\hline $\operatorname{EAS}(504)^{3}$ & $0.000^{¥}$ & $0.000^{\S}$ & $0.526^{¥ \S}$ & $0.923^{¥ \S}$ & $0.001^{*}$ & $0.000^{¥ \S}$ & $0.026^{¥}$ & $0.035^{¥ \S}$ & $0.202^{¥}$ \\
\hline SAS $(489)^{4}$ & $0.006^{¥}$ & $0.000^{\S}$ & $0.371^{¥}$ & $0.640^{¥ \S}$ & $0.004^{¥}$ & $0.000^{¥ \S}$ & $0.002^{\neq}$ & 0.325 & $0.090^{¥ \S}$ \\
\hline $\operatorname{AMR}(347)^{5}$ & 0.022 & 0.009 & $0.519^{¥ \S}$ & 0.461 & 0.007 & $0.078^{\S}$ & $0.052^{z}$ & 0.344 & $0.232^{¥}$ \\
\hline $\operatorname{SC}(373)^{6}$ & 0.030 & $\mathrm{nt}$ & 0.460 & $0.560^{¥ \S}$ & $\mathrm{nt}$ & $0.050^{¥ \S}$ & $0.030^{¥}$ & $\mathrm{nt}$ & 0.150 \\
\hline PR-POP1 $(251)^{7}$ & 0.043 & $\mathrm{nt}$ & $0.498^{¥ \S}$ & 0.436 & $\mathrm{nt}$ & $0.023^{¥ \S}$ & 0.022 & $\mathrm{nt}$ & $0.113^{¥}$ \\
\hline PR-POP2 $(400)^{8}$ & 0.028 & $\mathrm{nt}$ & $0.488^{¥ \S}$ & $0.365^{¥}$ & $\mathrm{nt}$ & $0.123^{¥ \S}$ & $\mathrm{nt}$ & $\mathrm{nt}$ & 0.151 \\
\hline SP $(948)^{9}$ & 0.024 & $\mathrm{nt}$ & $0.460^{\S}$ & $0.360^{¥}$ & 0.016 & $0.185^{¥ \S}$ & 0.020 & $0.279^{¥}$ & 0.150 \\
\hline $\mathrm{BA}(196)^{10}$ & 0.020 & $\mathrm{nt}$ & 0.380 & $0.357^{¥}$ & $\mathrm{nt}$ & $0.436^{¥}$ & 0.020 & $\mathrm{nt}$ & 0.110 \\
\hline $\operatorname{MG}(170)^{11}$ & 0.015 & $\mathrm{nt}$ & 0.406 & $0.297^{¥}$ & $\mathrm{nt}$ & $0.229^{¥ \S}$ & $\mathrm{nt}$ & $\mathrm{nt}$ & 0.126 \\
\hline
\end{tabular}

Data are presented as relative frequency. Euro-Brazilians and Afro-Brazilian, present study. ns, non-significant; nt, non-tested; ${ }^{¥} p$-value $<0.05$ when compared with Euro-Brazilians; ${ }^{\S} p$-value $<0.05$ when compared with Afro-Brazilians; ${ }^{\mathfrak{f}}$ statistical analysis for comparison between Afro-Brazilians and AFR cannot be performed.

${ }^{1}$ EUR: European (CEU, Utah Residents (CEPH) with Northern and Western Ancestry; TSI, Toscani in Italia; FIN, Finnish in Finland; GBR, British in England and Scotland; IBS, Iberian Population in Spain). ${ }^{2}$ AFR: African (YRI, Yoruba in Ibadan, Nigeria; LWK, Luhya in Webuye, Kenya; GWD, Gambian in Western Divisions in the Gambia; MSL, Mende in Sierra Leone; ESN, Esan in Nigeria; ASW, Americans of African Ancestry in the SW USA; ACB, African Caribbeans in Barbados). ${ }^{3}$ EAS: East Asian (CHB, Han Chinese in Beijing, China; JPT, Japanese in Tokyo, Japan; CHS, Southern Han Chinese; CDX, Chinese Dai in Xishuangbanna, China; KHV, Kinh in Ho Chi Minh City, Vietnam). ${ }^{4}$ SAS: South Asian (IH, Gujarati Indians from Houston, Texas; PJL, Punjabi from Lahore, Pakistan; BEB, Bengali from Bangladesh; STU, Sri Lankan Tamils from the UK; ITU, Indian Telugu from the UK). ${ }^{5}$ AMR: Admixed American (MXL, Mexican Ancestry from Los Angeles USA; PUR, Puerto Ricans from Puerto Rico; CLM, Colombians from Medellin, Colombia; PEL, Peruvians from Lima, Peru) of 1000 Genomes Project; ${ }^{6}$ SC: Blood donors from the state of Santa Catarina (Costa et al. (2016a and 2016b); ${ }^{7}$ PR-POP1: Blood donors from the Southwest region of the state of Parana (Zacarias et al. (2016), ${ }^{8}$ PR-POP2: Blood donors from the state of Parana (Guelsin et al. (2011), ${ }^{9}$ SP: Blood donors from the state of São Paulo (Ribeiro et al., 2009) ${ }_{1}^{10}$ BA: Admixed population from the state of Bahia (Costa et al., 2016a and 2016b and ${ }^{11}$ MG: Blood donors from the state of Minas Gerais (Alves et al., 2018). 
SC; $J K^{*} 02(p=0.013), \quad F Y^{*} 02 N .01(p<0.001)$, and $R H C E^{*} E(p=0.048)$ from PR-POP1; $J K^{*} 02(\mathrm{p}=0.016)$, $F Y^{*} 01(\mathrm{p}=0.003)$, and $F Y^{*} 02 N .01 \quad(p=0.004)$ from PRPOP2; $F Y^{*} 01(p<0.001), F Y^{*} 02 N .01(p<0.001)$, and $G Y P B^{*} S(p=0.047)$ from SP; and $F Y^{*} 01(p=0.005$ and $p<$ $0.001)$ and $F Y^{*} 02 N .01(p<0.001$ and $p<0.001)$ from BA and $M G$, respectively.

The allele frequencies for $K E L^{*} 06$ observed in AfroBrazilians were similar only in AMR (Table 2), though the $J K^{*} 02$ allele distribution in Afro-Brazilians was similar to that observed in the SAS, SC, BA and MG populations. Moreover, the $F Y^{*} 01$ allele frequency in Afro-Brazilians was similar to that in the EUR, AMR, PR, SP, BA and MG populations, but the $F Y^{*} 02 N .01$ allele frequency observed in the present sample was different in all populations, except for BA (Table 2). The frequency of the $D I^{*} 01$ variant was similar in all populations assessed. The $G Y P B * S$ variant showed different distributions in AFR $(p<0.001)$ and EAS $(p<0.001)$ and the $R H C E^{*} E$ variant in SAS $(p=0.028)$.

Pairwise $F_{\mathrm{ST}}$ values for the samples of the present study and the 1000 Genomes database are shown in Table 3. Low genetic distance $\left(F_{\mathrm{ST}}=0.055\right)$ between Euro- and Afro-Brazilians of Rio Grande do Sul was observed when evaluated for population differentiation. In relation to EUR populations, the Euro- and Afro-Brazilian groups showed the lowest $F_{\mathrm{ST}}$ values ( 0.004 and 0.080 , respectively), and lower $F_{\text {ST }}$ values were also observed in comparisons of our groups and AMR populations (0.009 for Euro- and 0.061 for Afro-Brazilians). In contrast, the highest genetic distance was found between Euro-Brazilians of Rio Grande do
Sul and the AFR population (0.431) and between AfroBrazilians and AFR (0.297). Figure 1 shows the genetic distance observed for the populations analyzed in this study based on blood group alleles. The main result of $F_{\mathrm{ST}}$ analysis indicated that the AFR population is genetically more distinct than the other populations. To evaluate the contribution of each variant to the genetic distance observed, $F_{S T}$ values were also estimated for each SNP by examining the present sample and the 1000 Genomes Database. According to $F_{\mathrm{ST}}$ values, rs2814778 (c.1-67T >C) and rs12075 $(c .125 A>G)$ polymorphisms in the ACKR1 gene (Duffy blood group) present high differentiation among populations (0.865 and 0.399 , respectively, Table 4$)$.

\section{Discussion}

We examined population differentiation for the distribution of blood group alleles in blood donors from Rio Grande do Sul. Analysis of all variants together demonstrated that Euro- and Afro-Brazilian individuals from Rio Grande do Sul are genetically close $\left(F_{S T}=0.055\right.$; Table 3$)$. However, when each locus was evaluated individually, $K E L^{*} 06$ and $F Y^{*} 02 N .01$ allele frequencies were found to be significantly higher in the Afro-Brazilian group when compared with the Euro-Brazilian group (Table 2). Furthermore, based on data for AFR and EUR populations of the 1000 Genomes database, $K E L^{*} 06$ allele frequencies differ between these continents, with higher frequencies in AFR than in EUR. The Fy and $\mathrm{Fy}^{\mathrm{b}}$ antigens of the Duffy blood group act as receptors for malarial parasites on human RBCs (Miller et al., 1976). The $F Y^{*} 02 N .01$ allele predominates in malaria-endemic areas, such as some Africa

Table 3 - Pairwise $F_{S T}$ among South Brazilian population and populations evaluated in 1000 Genome Database.

\begin{tabular}{|c|c|c|c|c|c|c|c|}
\hline & Euro-descendants & Afro-descendants & $\mathrm{AFR}^{1}$ & $\mathrm{EUR}^{2}$ & $\mathrm{EAS}^{3}$ & $\mathrm{SAS}^{4}$ & $\mathrm{AMR}^{5}$ \\
\hline Euro-descendants & - & & & & & & \\
\hline Afro-descendants & $\begin{array}{c}0.055 \\
(0.021-0.097)\end{array}$ & - & & & & & \\
\hline $\mathrm{AFR}^{1}$ & $\begin{array}{c}0.431 \\
(0.410-0.450)\end{array}$ & $\begin{array}{c}0.297 \\
(0.218-0.370)\end{array}$ & - & & & & \\
\hline $\mathrm{EAS}^{3}$ & $\begin{array}{c}0.200 \\
(0.178-0.222)\end{array}$ & $\begin{array}{c}0.350 \\
(0.274-0.415)\end{array}$ & $\begin{array}{c}0.633 \\
(0.621-0.646)\end{array}$ & $\begin{array}{c}0.205 \\
(0.186-0.224)\end{array}$ & - & & \\
\hline $\mathrm{SAS}^{4}$ & $\begin{array}{c}0.037 \\
(0.024-0.051)\end{array}$ & $\begin{array}{c}0.137 \\
(0.080-0.199)\end{array}$ & $\begin{array}{c}0.514 \\
(0.499-0.529)\end{array}$ & $\begin{array}{c}0.046 \\
(0.034-0.060)\end{array}$ & $\begin{array}{c}0.132 \\
(0.116-0.150)\end{array}$ & - & \\
\hline $\mathrm{AMR}^{5}$ & $\begin{array}{c}0.009 \\
(0.003-0.016)\end{array}$ & $\begin{array}{c}0.061 \\
(0.024-0.104)\end{array}$ & $\begin{array}{c}0.438 \\
(0.417-0.459)\end{array}$ & $\begin{array}{c}0.009 \\
(0.005-0.015)\end{array}$ & $\begin{array}{c}0.174 \\
(0.154-0.195)\end{array}$ & $\begin{array}{c}0.044 \\
(0.033-0.058)\end{array}$ & - \\
\hline
\end{tabular}

Data are presented as $F_{\mathrm{ST}}(95 \%$ confidence interval).

${ }^{1}$ AFR: African (YRI, Yoruba in Ibadan, Nigeria; LWK, Luhya in Webuye, Kenya; GWD, Gambian in Western Divisions in the Gambia; MSL, Mende in Sierra Leone; ESN, Esan in Nigeria; ASW, Americans of African Ancestry in the SW USA; ACB, African Caribbeans in Barbados). ${ }^{2}$ EUR: European (CEU, Utah Residents (CEPH) with Northern and Western Ancestry; TSI, Toscani in Italia; FIN, Finnish in Finland; GBR, British in England and Scotland; IBS, Iberian Population in Spain). ${ }^{3}$ EAS: East Asian (CHB, Han Chinese in Beijing, China; JPT, Japanese in Tokyo, Japan; CHS,Southern Han Chinese; CDX, Chinese Dai in Xishuangbanna, China; KHV, Kinh in Ho Chi Minh City, Vietnam). ${ }^{4}$ SAS: South Asian (IH, Gujarati Indians from Houston, Texas; PJL, Punjabi from Lahore, Pakistan; BEB, Bengali from Bangladesh; STU, Sri Lankan Tamils from the UK; ITU, Indian Telugu from the UK). ${ }^{5}$ AMR: Admixed American (MXL, Mexican Ancestry from Los Angeles USA; PUR, Puerto Ricans from Puerto Rico; CLM, Colombians from Medellin, Colombia; PEL, Peruvians from Lima, Peru). 


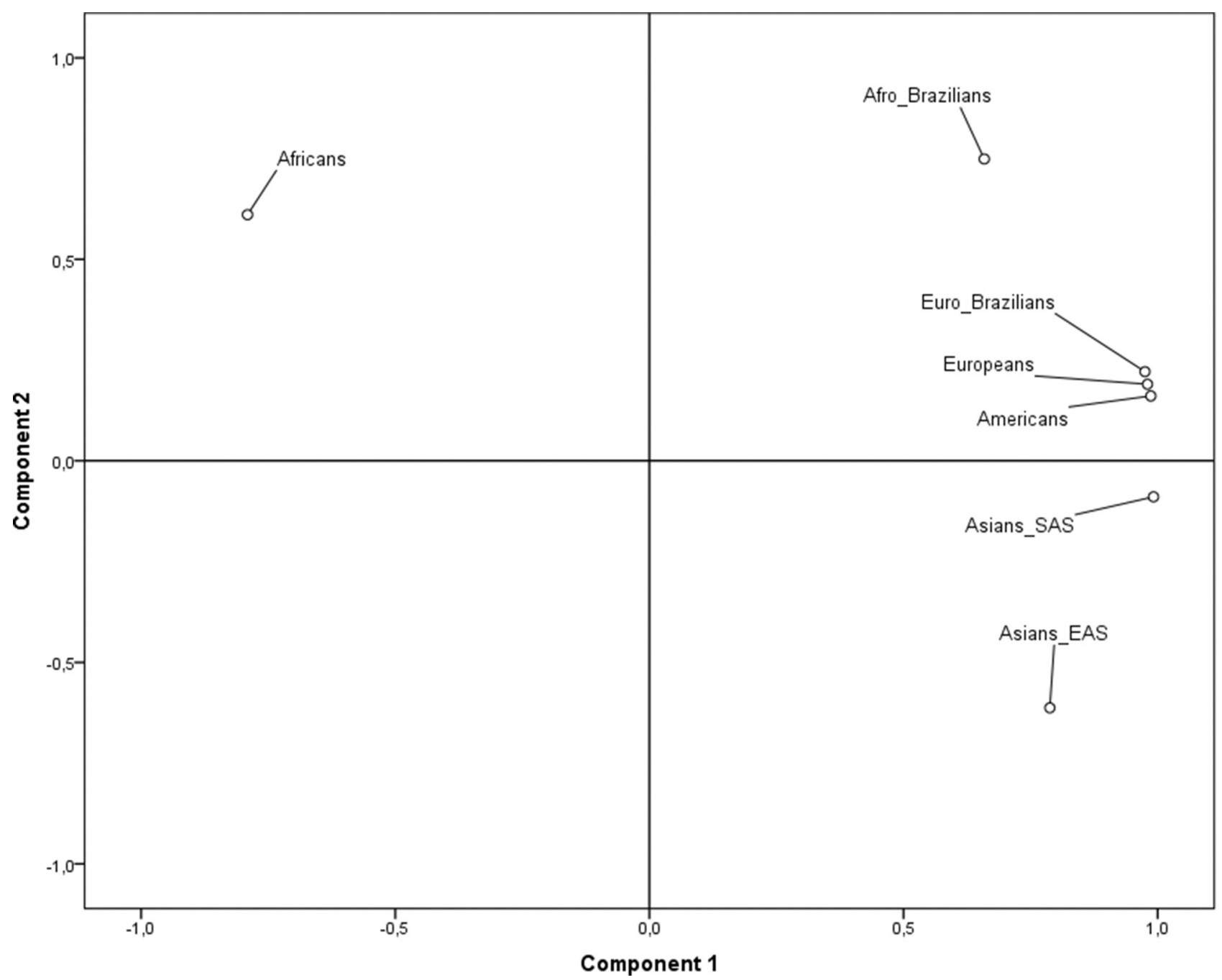

Figure 1 - Genetic distance among the populations analyzed in this study based on blood group alleles.

Table 4 - SNPs population differentiation.

\begin{tabular}{lcc}
\hline Gene & dbSNP & $F_{S T}(95 \% \mathrm{CI})$ \\
\hline$A C K R 1$ & $\mathrm{rs} 34599082$ & $0.007(0.003-0.012)$ \\
KEL & $\mathrm{rs} 8176058$ & $0.018(0.012-0.025)$ \\
SLC4A1 & $\mathrm{rs} 2285644$ & $0.028(0.018-0.040)$ \\
RHCE & $\mathrm{rs} 609320$ & $0.029(0.020-0.038)$ \\
SLC14A1 & $\mathrm{rs} 1058396$ & $0.064(0.051-0.079)$ \\
KEL & $\mathrm{rs} 8176038$ & $0.082(0.066-0.097)$ \\
GYPB & $\mathrm{rs} 7683365$ & $0.083(0.072-0.094)$ \\
$A C K R 1$ & $\mathrm{rs} 12075$ & $0.399(0.384-0.413)$ \\
$A C K R 1$ & $\mathrm{rs} 2814778$ & $0.865(0.847-0.881)$ \\
\hline
\end{tabular}

regions, because it prevents expression of the receptor on the erythrocyte membrane, and consequently, these erythrocytes become refractory to infection by malarial parasites. These antigenic determinants have been used for determining ethnic composition and as anthropological markers (Cavasini et al., 2007), possibly due to their impact on natural selection in different geographical regions (Miller et al., 1976; Hamblin et al., 2002). Taken together, these findings for $K E L * 06$ and $F Y^{*} 02 N .01$ allele frequencies distributions indicate, as expected, a greater African background in the genetic pool of Afro-Brazilians than in EuroBrazilians in Rio Grande do Sul.

Based on the comparison of allele frequencies, which was conducted separately for blood group systems, it is evident that our Euro-Brazilian group had more similarities with the EUR and AMR populations in the 1000 Genomes database than with the AFR, EAS and SAS populations (Table 2). Although some significant differences have been reported, the frequencies observed for these alleles were not highly discrepant among these populations. Moreover, these findings were corroborated by analyses of all genetic markers together (EUR: $F_{S T}=0.004$ and AMR: $F_{S T}=0.009$, Table 3). In the same way, the Afro-Brazilians were found to be closer to $\operatorname{EUR}\left(F_{S T}=0.080\right)$ and $\operatorname{AMR}\left(F_{S T}=0.061\right)$ than to $\operatorname{AFR}\left(F_{S T}=0.297\right)$ populations. It is important to emphasize that the classification of ancestry in our sample was performed according to phenotypic characteristics, as it is 
performed in the blood bank. Previous studies have also demonstrated a discrepancy between skin color information and genetic ancestry (Boquett et al., 2015; Lima-Costa et al., 2015). For example, Boquett et al. (2015) evaluated self-assessed skin color and HLA genetic information of bone marrow donors from the state of Rio Grande do Sul and found that Brazilian individuals self-assessing as Black were closer genetically to European populations than to African populations (Boquett et al., 2015). Lima-Costa et al. (2015) also demonstrated that the association between ethnoracial self-classification and genome-based ancestry is not linear.

Although the $K E L^{*} 06$ and $F Y^{*} 02 N .01$ allele frequencies indicated more African ancestry in the Afro-Brazilian group than in the Euro-Brazilian group, these allele frequencies in the former are intermediate between the AFR and EUR populations. These findings were expected due to the colonization process of southern Brazil, which is predominantly characterized by admixture between European descendants. Consequently, this population has a distinct genetic background in relation to populations from other Brazilian regions (Pena et al., 2011; Salzano and Sans, 2014).

When our data were compared to allele frequencies of blood donors from Santa Catarina (Costa et al., 2016a,b), Paraná (Guelsin et al., 2011; Zacarias et al., 2016), São Paulo (Ribeiro et al., 2009), Bahia (Costa et al., 2016a,b) and Minas Gerais (Alves et al., 2018), $J K^{*} 02, F Y^{*} 01$ and $F Y^{*} 02 N .01$ variants presented greater differences in frequency among Brazilian regions (Table 2). Regardless of the similarities in the ancestral process of colonization among some localities, European ancestry is uniformly preponderant in southern Brazil. For instance, in the Rio Grande do Sul population, the composition of Europeans, Africans, and Amerindians is $72.9 \%, 14 \%$, and $13 \%$, respectively. In Santa Catarina, it is $79.7 \%, 11.4 \%$, and $8.9 \%$, respectively. In the state of Paraná, the average individual has $71 \%$ European ancestry, followed by $17.5 \%$ African, and $11.5 \%$ Amerindian. In São Paulo, the genetic background of the population is composed of $62.9 \%$ European, $25.5 \%$ African, and $11.6 \%$ Amerindian. In Minas Gerais, it is $59.2 \%, 28.9 \%$, and $11.9 \%$, respectively (Manta et al., 2013). The genomic ancestry of the Bahia population is 42.4\% European, $50.5 \%$ African, and 5.8\% Amerindian (Lima-Costa et al., 2015). Despite observed interethnic genetic similarity, there are significant differences in the frequencies of RBC polymorphisms among these populations. This suggests that data must be well documented and considered within the perspective of transfusion medicine.

Although similarity was demonstrated between Euroand Afro-Brazilians when all variants were analyzed together, the ethnic classification that uses phenotypic criteria to find blood units with rare antigens may be important when the $K E L^{*} 06$ and, mainly, $F Y^{*} 02 N .01$ alleles are considered for this southern Brazilian population. Thus, when there is a need to detect blood units with an absence of Duffy antigens, there is a greater probability of finding donors in this group. To the best of our knowledge, no other studies have reported RBC genetic variability in Rio Grande do Sul, emphasizing the intense process of admixture that makes the Brazilian population unique in its ethnic background.

\section{Acknowledgments}

The authors are very grateful to Ananda C.S. Galvão (in memoriam) for her effort and dedication in this study. This study was supported by the grant PqG2014/FAPERGS 2373-2551/14-4.

\section{Conflict of Interest}

The authors declare that there is no conflict of interest that could be perceived as prejudicial to the impartiality of the reported research.

\section{Author Contribution}

$\mathrm{SA}, \mathrm{TO}$ and MF designed the research and conceived the project; $\mathrm{GW}$ and MMOR performed the data collection; GW, MMOR, GH, MF, and SA carried out the research; GW, MMOR, GH, MF, JDL, and SA performed the literature search and analyzed the data. GW, MMOR, GH, MF, and SA interpreted the data and wrote the manuscript. SA had the primary responsibility for the final content. All authors were involved in writing the paper and gave final approval to the submitted and published versions.

\section{References}

Alves VM, De Vito FB, Martins PRJ, Silva SS, Castilho L and Moraes-Souza H (2018) Frequency of red blood cell genotypes in multi-transfused patients and blood donors from Minas Gerais, Southeast Brazil. Transfus Apher Sci 57:71-75.

Anstee DJ (2009) Red cell genotyping and the future of pretransfusion testing. Blood 114:248-256.

Arnoni CP, Muniz JG, Paula TA, Person RDM, Gazito D, Baleotti Jr W, Barreto JA, Castilho L and Latini FRM (2013) An easy and efficient strategy for KEL genotyping in a multiethnic population. Rev Bras Hematol Hemoter 35:99-102.

Baleotti W, Suzuki RB, Polotto M, Ruiz MO, Fabron A and Castilho L (2011) A PCR-based strategy for Dombrock screening in Brazilian blood donors reveals a novel allele: The DO*A-WL. J Clin Lab Anal 25:79-82.

Boquett J, Schüler-Faccini L, Jobim LF, Jobim M, Fagundes NJR and Hünemeier T (2015) Self-assessment of color categories and its relationship with HLA profiling in Brazilian bone marrow donors. Biol Blood Marrow Transplant 21:1140-1144.

Cavasini CE, De Mattos LC, Couto AARDA, Couto VSCDA, Gollino Y, Moretti LJ, Bonini-Domingos CR, Rossit ARB, Castilho L and Machado RLD (2007) Duffy blood group gene polymorphisms among malaria vivax patients in four areas of the Brazilian Amazon region. Malar J 6:1-8. 
Costa D, Schinaider A, Santos M, Schörner E, Simon D, Maluf S, Moraes A and Silva M (2016a) Frequencies of polymorphisms of the Rh, Kell, Kidd, Duffy and Diego systems of Santa Catarina, Southern Brazil. Rev Bras Hematol Hemoter 38:199-205.

Costa DC, Schinaider AA, Santos TM, Schörner EJ, Simon D, Maluf SW, Moraes ACR and Silva MCS (2016b) Erratum to "Frequencies of polymorphisms of Rh, Kell, Kidd, Duffy and Diego systems of Santa Catarina, southern Brazil”. Rev Bras Hematol Hemoter 38:371-372.

Credidio D, Pellegrin J and Castilho L (2011) Serologic and molecular characterization of D variants in Brazilians: Impact for typing and transfusion strategy. Immunohematology 27:6-11.

Cruz BR, Chiba AK, Moritz E and Bordin JO (2012) RHD alleles in Brazilian blood donors with weak D or D-negative phenotypes. Transfus Med 22:84-89.

Durso DF, Bydlowski SP, Hutz MH, Suarez-Kurtz G, Magalhães TR and Pena SDJ (2014) Association of genetic variants with self-assessed color categories in Brazilians. PLoS One 9:e83926.

Excoffier L and Lischer HEL (2010) Arlequin suite ver 3.5: A new series of programs to perform population genetics analyses under Linux and Windows. Mol Ecol Resour 10:564-567.

Faria MA, Martins ML, Schmidt LC and Malta MCFS (2012) Molecular analysis of the GYPB gene to infer S, s, and U phenotypes in an admixed population of Minas Gerais, Brazil. Rev Bras Hematol Hemoter 34:212-216.

Flôres MALR, Visentainer JEL, Guelsin GAS, Fracasso AS, Melo FC, Hashimoto MN and Sell AM (2014) Rh, Kell, Duffy, Kidd and Diego blood group system polymorphism in Brazilian Japanese descendants. Transfus Apher Sci 50:123-128.

Fox J (2005) The R commander: A basic statistics graphical user interface to R. J Stat Softw 14:1-42.

Guelsin GAS, Sell AM, Castilho L, Masaki VL, Melo FC, Hashimoto MN, Hirle LS and Visentainer JEL (2011) Genetic polymorphisms of Rh, Kell, Duffy and Kidd systems in a population from the State of Paraná, southern Brazil. Rev Bras Hematol Hemoter 33:21-5.

Hamblin MT, Thompson EE and Di Rienzo A (2002) Complex signatures of natural selection at the Duffy blood group locus. Am J Hum Genet 70:369-383.

Issitt P and Anstee D (1998) Applied Blood Group Serology. 4th edition. Montgomery Scientific Publications, Montgomery, $1232 \mathrm{pp}$.

Keenan K and McGinnity P (2013) diveRsity: An R package for the estimation of population genetics parameters and their associated errors. Methods Ecol Evol 4:782-788.

Lahiri D and Nurnberger J (1991) A rapid non-enzymatic method for the preparation of HMW DNA from blood for RFLP studies. Nucleic Acids Res 19:5444.

Lima-Costa M, Rodrigues L, Barreto M, Gouveia M, Horta B, Mambrini J, Kehdy F, Pereira A, Rodrigues-Soares F, Victora $\mathrm{C}$ et al. (2015) Genomic ancestry and ethnoracial selfclassification based on 5,871 community-dwelling Brazilians (The Epigen Initiative). Sci Rep 5:9812.

Manta FSN, Pereira R, Vianna R, Araújo A, Gitaí D, Silva D, Wolfgramm E, Pontes I, Aguiar J, Moraes M et al. (2013)
Revisiting the genetic ancestry of Brazilians using autosomal AIM-indels. PLoS One 8:e75145.

Miller LH, Mason SJ, Clyde DF and McGinniss MH (1976) The resistance factor to Plasmodium vivax in blacks - the Duffyblood-group genotype, FyFy. N Engl J Med 295:302-304.

Mota M, Dezan M, Valgueiro MC, Sakashita AM, Kutner JM and Castilho L (2012) RHD allelic identification among D-Brazilian blood donors as a routine test using pools of DNA. J Clin Lab Anal 26:104-108.

Parra FC, Amado RC, Lambertucci JR, Rocha J, Antunes CM and Pena SDJ (2003) Color and genomic ancestry in Brazilians. Proc Natl Acad Sci U S A 100:177-182.

Pellegrino J, Castilho L, Rios M and De Souza CA (2001) Blood group genotyping in a population of highly diverse ancestry. J Clin Lab Anal 15:8-13.

Pena SDJ, di Pietro G, Fuchshuber-Moraes M, Genro JP, Hutz MH, Kehdy FSG, Kohlrausch F, Magno LAV, Montenegro RC, Moraes MO et al. (2011) The genomic ancestry of individuals from different geographical regions of Brazil is more uniform than expected. PLoS One 6:e17063.

Piassi FCC, Santos SME, Castilho LM, Baleotti Júnior W, Suzuki RB and Cunha DM (2013) Dombrock genotyping in Brazilian blood donors reveals different regional frequencies of the HY allele. Rev Bras Hematol Hemoter 35:400-403.

Ribeiro KR, Guarnieri MH, Da Costa DC, Costa FF, Pellegrino J and Castilho L (2009) DNA array analysis for red blood cell antigens facilitates the transfusion support with antigenmatched blood in patients with sickle cell disease. Vox Sang 97:147-152.

Salzano FM and Sans M (2014) Interethnic admixture and the evolution of Latin American populations. Genet Mol Biol 37:151-170.

Santos SA (2002) Historical roots of the "Whitening" of Brazil. Polit Cult Form 29:61-82.

Zacarias JMV, Langer IBV, Visentainer JEL and Sell AM (2016) Profile of Rh, Kell, Duffy, Kidd, and Diego blood group systems among blood donors in the Southwest region of the Paraná state, Southern Brazil. Transfus Apher Sci 55:302-307.

\section{Internet resources}

Instituto Brasileiro de Geografia e Estatística I (2017) Área territorial oficial, http://www.ibge.gov.br/home/geociencias/cartografia/default_territ_area.shtm (accessed 25 February 2017).

International Society of Blood Transfusion I (2017) Red Cell Immunogenetics and Blood Terminology, http://www.isbtweb.org/working-parties/red-cellimmunogenetics-and-blood-group-terminology (accessed 25 February 2017).

ThermoFisher Scientific SNP Genotyping Analysis Using TaqMan Assays, https://www.thermofisher.com/br/en/home/life-science/pcr /real-time-pcr/real-time-pcr-assays/snp-genotyping-taqman -assayshtml.

Associate Editor: Regina C. Mingroni-Netto

License information: This is an open-access article distributed under the terms of the Creative Commons Attribution License (type CC-BY), which permits unrestricted use, distribution and reproduction in any medium, provided the original article is properly cited. 